\title{
Light Element Abundances Constrain Primordial Black Holes in the Critical Collapse Model
}

\author{
Chao Chen ${ }^{1, *}$, Yudong Luo ${ }^{2,3, * *}$, Motohiko Kusakabe ${ }^{4, * *}$, and Toshitaka Kajino ${ }^{2,3,4, * * * *}$ \\ ${ }^{1}$ Jockey Club Institute for Advanced Study, The Hong Kong University of Science and Technology, \\ Hong Kong S.A.R., China \\ ${ }^{2}$ Graduate School of Science, The University of Tokyo, 7-3-1 Hongo, Bunkyo-ku, Tokyo 113-0033, \\ Japan \\ ${ }^{3}$ National Astronomical Observatory of Japan, 2-21-1 Osawa, Mitaka, Tokyo 181-8588, Japan \\ ${ }^{4}$ School of Physics, International Research Center for Big-Bang Cosmology and Element Genesis, Bei- \\ hang University, 37, Xueyuan Rd., Haidian-qu, Beijing 100083 China
}

\begin{abstract}
We study the photodisintegration process triggered by the nonthermal electromagnetic Hawking radiation from primordial black holes (PBHs) in the critical collapse model. The presence of a low-mass tail of critical collapse mass function could enhance energetic photon emissions from Hawking radiation of PBHs. Nuclear photodisintegration rates are calculated with a nonthermal photon spectrum derived by solving the Boltzmann equation iteratively. With the newest observational limit on the ${ }^{3} \mathrm{He}$ abundance in Galactic $\mathrm{H}$ II regions, the updated ${ }^{3} \mathrm{He}$ constraints on $\mathrm{PBH}$ mass spectrum in the horizon mass range $10^{12}-10^{13} \mathrm{~g}$ are derived. Our results show that ${ }^{3} \mathrm{He}$ constraints on the critical mass function are about one order of magnitude severer than the monochromatic one, although the fraction of PBHs in the low-mass tail region is relatively small.
\end{abstract}

\section{Introduction}

Primordial black holes (PBHs) may be formed from density fluctuations in the very early Universe and have been studied over more than 50 years [1,2]. PBHs could be small enough for Hawking radiation to be observationally significant [3]. It has been well understood that the mass distribution of PBHs is extended in many scenarios [4]. Even for PBHs forming at a single epoch, they would have an extended mass distribution when critical phenomena of gravitational collapse are taken into account [5]. We show in this paper that the small amounts of PBHs within the low-mass tail in the mass function of the critical collapse model would affect the elemental abundances. More stringent BBN constraints are then acquired than those on the monochromatic one due to the simple fact that the Hawking radiation in the high energy region is stronger for low-mass PBHs. This leads to a caution that other constraints for the critical collapse model may also be altered compared to the monochromatic case.

\footnotetext{
*e-mail: iascchao@ust.hk

**e-mail: yudong.luo@nao.ac.jp

***e-mail: kusakabe@buaa.edu.cn

****e-mail: kajino@buaa.edu.cn
} 


\section{Critical Collapse Mass Function and Nonthermal Nucleosynthesis via Hawking Radiation}

It is well known that PBH formation is associated with the critical phenomena of gravitational collapse, and the early studies showed that the PBH mass at the formation epoch has a universal scaling property [6]

$$
M=K M_{H}\left(\delta-\delta_{c}\right)^{v},
$$

near the threshold $\delta \simeq \delta_{c}$ for $\mathrm{PBH}$ formation. The rescaling relation between $\mathrm{PBH}$ masses $M$ and density contrast $\delta$ implies that the $\mathrm{PBH}$ masses are naturally extended due to the Gaussian distribution of $\delta$, the differential comoving number density is given by

$$
n(M)=\frac{1}{\beta} n_{\mathrm{PBH}}\left(t_{f}\right) P[\delta(M)] \frac{d \delta(M)}{d M},
$$

for each horizon mass $M_{H}, \beta$ (or the redefined $\beta^{\prime}$ in the following part) is the initial PBH abundances. Considering PBHs with an extended mass function, the total Hawking emission should be contributed by all individual mass scales. Figure 1 plots the instantaneous emission

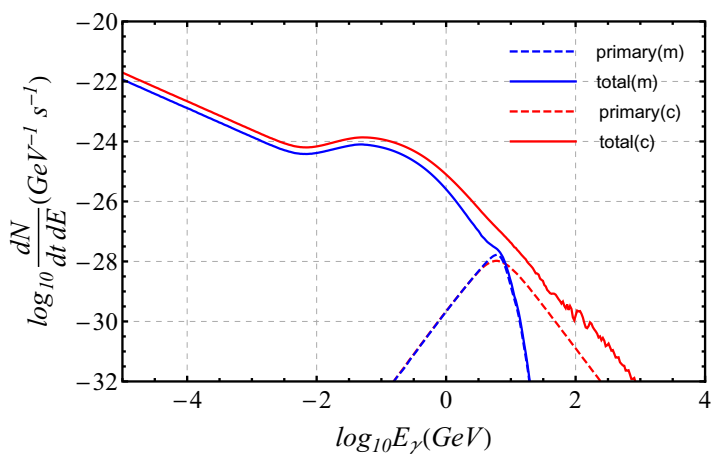

Figure 1. Instantaneous emission rate of photons for PBHs with horizon mass $10^{13} \mathrm{~g}$, for the same energy fraction of the monochromatic (denoted by the script " $m$ ", the blue curve) and critical collapse mass functions (denoted by the script "c", the red curve).

rate of photons for PBHs with the horizon mass $10^{13} \mathrm{~g}$ for monochromatic (blue) and critical collapse mass functions (red) at the formation epoch, in which we set the total number density of PBHs with these two types of mass functions to be equal.

In this study, we mainly focus on PBHs within horizon mass $10^{12} \mathrm{~g} \leq M_{\mathrm{H}} \leq 10^{13} \mathrm{~g}$ and their lifetime $10^{8} \mathrm{~s} \leq \tau \leq 10^{13} \mathrm{~s}$. For PBHs within this range, proton and neutron emissions contribute to $<20 \%$ of the final production [12] so that the hadrodissociation process could not play a dominant role, the most important process is the photodisintegration triggered by non-thermal photons. The injections of energetic photons via Hawking radiation would initiate the electromagnetic (EM) cascade showers which are mainly made of energetic photons and pairs of electron and positron. At different cosmic temperature $T$, the photons would form a quasi-static equilibrium spectrum $f_{\gamma}\left(E_{\gamma} ; T\right)$ very quickly, and $f_{\gamma}\left(E_{\gamma} ; T\right)$ is given by the solution of the steady Boltzmann equation:

$$
\begin{aligned}
\frac{\partial f_{\gamma}\left(E_{\gamma} ; T\right)}{\partial t}=\frac{\partial}{\partial E}[ & \left.b_{\exp }(E ; T) f_{\gamma}\left(E_{\gamma} ; T\right)\right] \\
& -\Gamma_{\gamma}\left(E_{\gamma} ; T\right) f_{\gamma}\left(E_{\gamma} ; T\right)+S\left(E_{\gamma} ; T\right) \equiv 0 .
\end{aligned}
$$


Here, $S\left(E_{\gamma} ; T\right)$ is the source term that depends on the energies of the Hawking-radiated photons at a cosmic temperature $T$, i.e., the time evolution of Hawking spectrum in terms of $T$. $b_{\text {exp }}(E ; T)$ is the photon energy loss rate via the cosmic expansion, and $\Gamma_{\gamma}\left(E_{\gamma} ; T\right)$ describes the degradation rate of zeroth generation nonthermal photons, respectively. The photodisintegration of nuclei can be triggered by photons with energies larger than the photodisintegration energy threshold. The time evolution of the nuclear abundances is governed by

$$
\frac{d Y_{A}}{d t}=\sum_{T} N_{A C}[T \gamma]_{A} Y_{T}(T)-\sum_{P}[A \gamma]_{P} Y_{A}(T),
$$

where $[T \gamma]_{A}$ and $[A \gamma]_{P}$ are producing and destroying reaction rates for nuclei $A$ of reactions $\gamma+T \rightarrow A$ and $\gamma+A \rightarrow P$, respectively. $N_{A C}$ is the number of the identical nuclear species in the final state. Secondary nonthermal reactions also occur if products of the primary photodisintegration reactions are energetic enough.

\section{Light Element Abundance Constraints on PBHs}

We use a nonthermal BBN nuclear reaction network code and have updated the reaction rates of nuclei with mass numbers $A \leq 10$ using the JINA REACLIB Database [8]. For the nonthermal photodisintegration reaction rates, we take into account both the primary and the secondary photodisintegration reactions. We run the nonthermal $\mathrm{BBN}$ code to put constraints

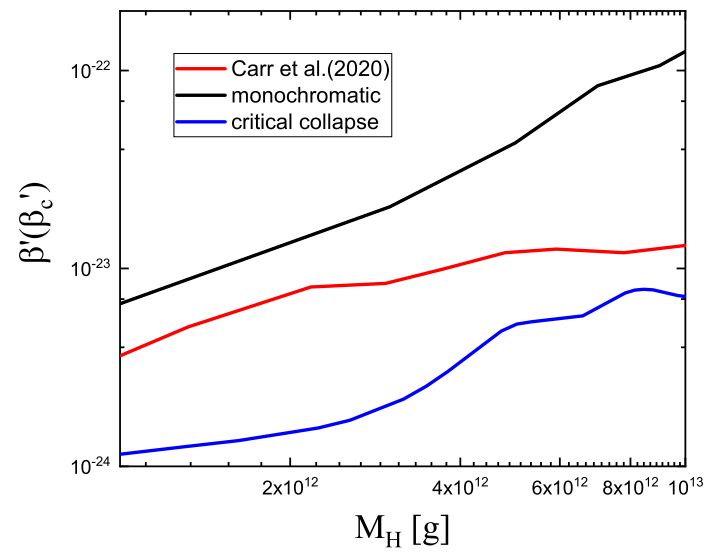

Figure 2. The upper bounds on $\beta^{\prime}\left(M_{H}\right)$ (the black curve) and $\beta_{c}^{\prime}\left(M_{H}\right)$ (the blue curve) in the horizon mass range $M_{H}=10^{12}-10^{13} \mathrm{~g}$ for the monochromatic and critical collapse mass functions, respectively, from the ${ }^{3} \mathrm{He} / \mathrm{H}$ ratio in the present Galaxy. The red curve refers to the constraint from Carr et al. [9].

on the initial mass spectrum for PBHs within the horizon mass range $10^{12}-10^{13} \mathrm{~g}$. By using the ${ }^{3} \mathrm{He}$ abundance, we plot the upper limits of $\beta^{\prime}\left(M_{H}\right)$ and $\beta_{c}^{\prime}\left(M_{H}\right)$ for the monochromatic and critical collapse mass functions in figure 2, respectively. The black curve shows our updated constraints on $\beta^{\prime}$ for the monochromatic mass function of PBHs within mass range $10^{12}-10^{13} \mathrm{~g}$ by using the observational limit ${ }^{3} \mathrm{He} / \mathrm{H}<1.5 \times 10^{-5}$. Compared with the constraint in Ref. [9] (the red curve), there exits one order of magnitude difference between our constraints and theirs. Considering that they used the more conservative constraint ${ }^{3} \mathrm{He} / \mathrm{D}<1.37$, the constraint derived in this study is much weaker. The hadrodissociation is ignored in this work, however, for the realistic PBH evaporation for this mass range, the 
branching ratio of proton energy normalized to the total available mass of $\mathrm{PBH}$ is less than $20 \%$. Therefore, even considering the hadrodissociation process in our calculation, our result could only move down utmost by half an order of magnitude, which still cannot explain the present inconsistency. The analytical expressions of the ${ }^{3} \mathrm{He}$ constraints on $\beta^{\prime}$ including both $100 \%$ photodisintegration and $100 \%$ hadrodissociation, are performed in Ref. [13], the estimated bounds should be the conceivably strongest, our result is consistent with the analytical estimations. We also see that the constraint on $\beta_{c}^{\prime}\left(M_{H}\right)$ (blue curve) is lower than that of the monochromatic one due to the enhancement of high-energy photons from the low-mass tail.

\section{Conclusion}

This work studies the nuclear photodisintegration reactions triggered by nonthermal electromagnetic Hawking radiation from PBHs in the critical collapse model, with the initial horizon masses of $10^{12}-10^{13} \mathrm{~g}$. As a consequence of Hawking radiation, the low-mass tail of the critical collapse mass function would enhance high-energy photon emission compared with the monochromatic mass function. We find out in this work that the high-energy photons radiated from the low-mass tail of the critical collapse mass function could significantly affect the primordial ${ }^{3} \mathrm{He}$ abundances via nonthermal BBN processes. We update the constraint on the initial mass spectrum $\beta^{\prime}$ of PBHs in the monochromatic and critical collapse model, respectively. Our result indicates that the tail contribution of the critical collapse model should be referred to with caution when considering the constraints on $\mathrm{PBH}$ evaporation.

Y.L. is supported by JSPS KAKENHI Grant No. 19J22167. M. K. is supported by NSFC Research Fund for International Young Scientists (11850410441). T. K. is supported in part by Grants-in-Aid for Scientific Research of JSPS (17K05457 and 20K03958).

\section{References}

[1] Y. B. Zel'dovich and I. D. Novikov, Sov. Astron. 10, 602 (1967).

[2] S. Hawking, Mon. Not. Roy. Astron. Soc. 152, 75 (1971).

[3] S. W. Hawking, Nature 248, 30 (1974).

[4] B. Carr, F. Kuhnel and M. Sandstad, Phys. Rev. D 94, no. 8, 083504 (2016).

[5] M. W. Choptuik, Phys. Rev. Lett. 70, 9-12 (1993).

[6] J. C. Niemeyer and K. Jedamzik, Phys. Rev. Lett. 80, 5481-5484 (1998).

[7] M. Kawasaki, K. Kohri and T. Moroi, Phys. Rev. D 63, 103502 (2001).

[8] P. Descouvemont, A. Adahchour, C. Angulo, A. Coc and E. Vangioni-Flam, Atom. Data Nucl. Data Tabl. 88, 203-236 (2004).

[9] B. Carr, K. Kohri, Y. Sendouda and J. Yokoyama, [arXiv:2002.12778 [astro-ph.CO]].

[10] M. Kusakabe, A. B. Balantekin, T. Kajino and Y. Pehlivan, Phys. Rev. D 87, no.8, 085045 (2013).

[11] H. Ishida, M. Kusakabe and H. Okada, Phys. Rev. D 90, no.8, 083519 (2014).

[12] J. H. MacGibbon, Phys. Rev. D 44, 376-392 (1991).

[13] Y. Luo, C. Chen, M. Kusakabe and T. Kajino, J. Cosmol. Astropart. Phys. 05, 042 (2021). 